\title{
Opportunism Transmit Mechanism Research in Cognitive Network
}

\author{
Xueting Niu \\ Dongchang College of LiaoCheng University, Liaocheng252000, China \\ xueting167@163.com
}

\begin{abstract}
Under the condition of limited feedback, due to the multiple antennas cognitive two-way relay system due to the relay node have been the channel state information and the actual channel state information is error, caused the user system interference to primary user system increases, at the same time reduces the transmission rate of user system. Aiming at this problem, this paper on the basis of the statistical features of quantization error, put forward an algorithm to overcome the quantization error of beam forming. This algorithm under the condition of limited interference to primary user system, with the minimum mean square error (mse) is a standard, improved the user's transmission rate, the simulation results show that the proposed algorithm compared with other algorithm, effectively suppress the influence of quantization error, improve the transmission rate of user system, reduces the error rate of user system.
\end{abstract}

Keywords: Cognitive networks, two-way relay, beam forming, simulation

\section{Introduction}

Spectrum shortage has been the bottleneck of wireless communication development, in order to overcome this problem, many methods have been proposed. Among them, Cognitive Radio technology is widely attention in recent years [1]. The essence of the cognitive radio technology is dynamically allocated and idle spectrum in order to improve the spectrum utilization. At the same time, the multi-antenna technology in order to make full use of space resources, can exponentially improve system capacity and the focal point of the wireless communication technology [2-4]. In order to solve the problem of system, relay technology obtained the widespread attention. In the relay technology, two-way relay technology with its high transmission efficiency, confidentiality good performance becomes the object of extensive research [5]. Cognitive radio system in the process of transmission, the main challenge is the primary user system's Quality of Service, at the same time, how to improve the transmission rate of user system. Multiple antenna two-way relay set by the user system, can play the advantages of multiple antennas to improve system capacity, little interference to other systems can also play a relay technology, the advantages of two-way relay technology to ensure the confidentiality of user's system, become the most practical transmission system [6-8].

In this system, many studies have been started. Two-way relay[9] to study the single antenna beam forming algorithm of cognitive system, document [10] to study the multiple antenna beam forming algorithm in two-way relay cognitive system and power allocation method, the algorithm under the condition of guarantee the QoS of primary user system, the maximum transmission rate times the user's system [11].under the multiple users on a two-way relay cognitive system design of beam forming algorithm, 
under the multiple users on the literature [12] two-way relay of beam forming algorithm of cognitive system security features. However the above literature research are in relay node for full Channel State Information (the Channel State Information, the CSI) conditions. In the actual frequency division duplex system, the relay node to complete CSI need high system cost, so the limited feedback method is proposed, for quantification of channel state information. Compared to these quantitative CSI and complete CSI, quantization error, if you don't change the beam forming algorithm, and can cause interference increases, primary user system and reduces the transmission rate of user system. The work of this paper is based on the statistical properties of the quantization error of the design of beam forming algorithm, to reduce the negative influence of quantization error. In this paper, based on minimum mean square error to optimize the design of the standard beam forming algorithm, simulation results show that the proposed scheme is compared with the existing schemes, the user system capacity and the bit error performance have great ascension.

\section{System Model}

System model of the proposed scheme is shown in Figure 1, the system includes a pair of primary user transceiver, $\boldsymbol{K}$ pairs of second users transceiver. Each pair of users in need through the same Relay (Relay Station, RS) for information exchange, primary user has $N$ receiving antenna, each second users has $N$ antenna, RS has $M$ antenna.

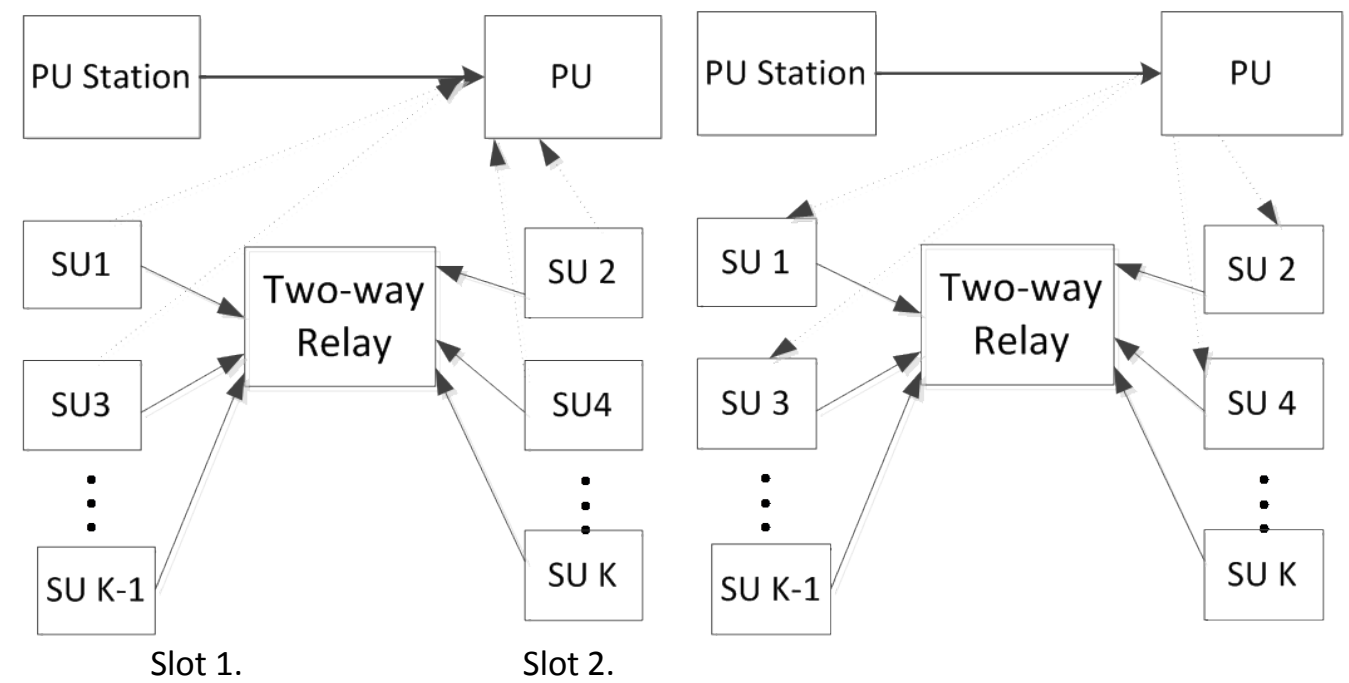

Figure 1. Cognitive Two-Way Relay System

At the first time slot, all second usesr sends the message to the two-way relay. In the second time slot, two-way relay will sent the signals which have been processing t to the second users. The dotted line represents the interference to primary user. Without loss of generality, assuming that there is no direct communication link between the second users. This system is frequency division duplex system, in the first time slot, second user $i(i=1, \cdots, K)$ send the signal $\mathbf{S}_{i}$ to the two-way relay station, so the relay station receives the signal can be expressed as

$$
\mathbf{y}_{R}=\sum_{i=1}^{K} \sqrt{P_{i}} \mathbf{H}_{i} \mathbf{S}_{i}+\mathbf{n}_{R}
$$


Where $\mathbf{S}_{i} \in \square^{N \times 1}$ is the signal which the second user $i$ send, due to the power normalization, so $E\left(\mathbf{S}_{i} \mathbf{S}_{i}^{\mathrm{H}}\right)=\mathbf{I}_{N} \cdot \square^{N \times M}$ denotes matrix element is the plural, and The matrix is $N$ row $M$ column. $\mathbf{I}_{N}$ denotes $N \times N$ identity matrix. $E(\bullet)$ is on behalf of the averaging operation. $(\bullet)^{\mathrm{H}}$ denotes the conjugate transpose of matrix operation. $\mathbf{H}_{i} \in \square^{M \times N}$ is the channel between the second user and two-way relay. Assuming the channel obey Rayleigh fading channel. $\sqrt{P_{i}}$ is the power of the second user ${ }^{i}$ transmitting in the first time slot. $\mathbf{n}_{R} \in \square^{M \times 1}$ is the noise that relay terminal receiving. This paper analyses the situation that additive white gaussian noise, the mean to 0 , noise variance can be expressed as $E\left(\mathbf{n}_{R} \mathbf{n}_{R}^{\mathrm{H}}\right)=\sigma^{2} \mathbf{I}_{M}$. Because this article studies the beam forming algorithm under the condition of limited feedback, so set the user's power with the method of average distribution. Because of the second user and the primary users in the same frequencies for transmission, from the second user sends a signal will reach the primary user, thus causing interference to primary user. The primary user receives the disturbance signal can be expressed as

$$
\mathbf{Z}_{1}=\sum_{i=1}^{K} \sqrt{P_{i}} \mathbf{G}_{i} \mathbf{S}_{i}+\mathbf{v}_{1}
$$

Where is in the primary user of additive white gaussian noise, the mean to 0 , the variance is $\sigma^{2}, \mathbf{G}_{i}$ is the channel between PU and SU, $\mathbf{G}_{i} \in \square^{M \times N}$. In order to ensure that the user's normal communication, SU interference to primary user cannot exceed a specific value, so denote

$$
\sum_{i=1}^{K}\left\|\mathbf{G}_{i}\right\|^{2} P_{i} \leq \eta
$$

In two-way relay, receiving signals sent through linear beam forming algorithm, then treated with beam forming algorithm of signal can be expressed as

$$
\mathbf{X}=\mathbf{F} \mathbf{y}_{R}
$$

Where $\mathbf{F} \in \square^{N \times N}$ is the beamforming matrix of relay station, in order to second users on the receiving signal to eliminate the multi-user interference between each other. In addition, the limitation on the relay side has power $P_{R}$, namely the sending power is no more than rated value, can be expressed as

$$
\operatorname{Tr}\left(\sum_{i=1}^{K} \mathbf{F} \mathbf{H}_{i} \mathbf{H}_{i}^{\mathrm{H}} \mathbf{F}^{\mathrm{H}} P_{i}+\mathbf{F} \mathbf{F}^{\mathrm{H}} \sigma^{2}\right) \leq P_{R}
$$

Among them, $\operatorname{Tr}(\bullet)$ is on behalf of the matrix of the trace operation.

At the second time slot, namely the broadcast phase, a relay node to beam forming processing signal sent out, the user receives the signal can be written as

$$
\mathbf{y}_{i}=\mathbf{H}_{i}^{\mathrm{H}} \mathbf{X}+\mathbf{n}_{i}
$$

At SU the receiving beam forming matrix, to eliminate multiuser interference. 
Among them $\mathbf{n}_{i}$ is the additive white gaussian noise, the mean to 0 , the variance is $\sigma^{2}$. At this point by the relay radio signals interference to the primary user is also less than a specific value $\eta$, i.e.,

$$
\operatorname{Tr}\left(\sum_{i=1}^{K} \mathbf{G}_{i} \mathbf{F} \mathbf{H}_{i} \mathbf{H}_{i}^{\mathrm{H}} \mathbf{F}^{\mathrm{H}} \mathbf{G}_{i}^{\mathrm{H}} P_{i}+\mathbf{G}_{i} \mathbf{F} \mathbf{F}^{\mathrm{H}} \mathbf{G}_{i}^{\mathrm{H}} \sigma^{2}\right) \leq \eta
$$

\section{Feedback Scheme}

$\mathbf{H}_{i}^{\mathrm{H}} \in \square^{N \times M}$ are the channel from relay node to SU, and the element is subject to plural independent identically distributed gaussian variables, the mean to 0 , the variance is 1 , in this paper, the author studies on feedback, the code is built on the fixed quantity, fixed number of bits in the feedback channel, and relay the client and client are set in advance. In code C elements contained $2^{\mathrm{B}}$ in this set, and each element $\square^{N \times M}$ matrix, therefore, to use $\left(\mathbf{W}_{1}, \ldots, \mathbf{W}_{2^{B}}\right)$, and $B$ is each time the user back to the number of bits in the relay.

This article uses the literature [13] quantization scheme, this scheme adopts the random code, random code of this scheme can achieve the optimal performance; In the option code according to the following standards

$$
\hat{\mathbf{H}}_{i}=\underset{\mathbf{w} \in \mathrm{C}}{\arg \min } d^{2}\left(\mathbf{H}_{i}, \mathbf{W}\right)
$$

Which $d\left(\mathbf{H}_{i}, \mathbf{W}\right)$ represent and different between $\mathbf{H}_{i}$ and $\mathbf{W}$ various elements, this paper uses the string distance (choral short) in order to solve the above problem, in order to facilitate analysis, this article uses the literature [13] decomposition approach:

$$
\tilde{\mathbf{H}}_{i}=\hat{\mathbf{H}}_{i} \mathbf{X}_{i} \mathbf{Y}_{i}+\mathbf{S}_{i} \mathbf{Z}_{i}
$$

$\mathbf{S}_{i}$ is on behalf of the code in this space of null space, said the normalized projection in value; According to its statistical properties, according to the literature [13]

$$
\begin{gathered}
\mathbf{Y}_{i}^{\mathrm{H}} \mathbf{Y}_{i}=\tilde{\mathbf{H}}_{i}^{\mathrm{H}} \mathbf{W} \mathbf{W}^{\mathrm{H}} \tilde{\mathbf{H}}_{i} \\
\operatorname{Tr}\left(\mathbf{Y}_{i}^{\mathrm{H}} \mathbf{Y}_{i}\right)=N-d^{2}\left(\tilde{\mathbf{H}}_{i}, \mathbf{W}\right) \\
\mathbf{Z}_{k}^{\mathrm{H}} \mathbf{Z}_{k}=\mathbf{I}_{N}-\mathbf{Y}_{k}^{\mathrm{H}} \mathbf{Y}_{k} \\
\operatorname{Tr}\left(\mathbf{Z}_{i}^{\mathrm{H}} \mathbf{Z}_{i}\right)=d^{2}\left(\tilde{\mathbf{H}}_{i}, \mathbf{W}\right)=D
\end{gathered}
$$

Made $\mathbf{H}_{i} \mathbf{H}_{i}^{\mathrm{H}}=\tilde{\mathbf{H}}_{i} \boldsymbol{\Lambda}_{i} \tilde{\mathbf{H}}_{i}^{H}, \boldsymbol{\Lambda}_{i}=\operatorname{diag}\left[\lambda_{1}, \cdots, \lambda_{N}\right]$ is the decomposition of characteristic matrix $\mathbf{H}_{i} \mathbf{H}_{i}^{\mathrm{H}}$. Statistical properties [14] so it can get the following:

$$
\begin{gathered}
\mathrm{E}\left\{\mathbf{X}_{i} \mathbf{Y}_{i} \boldsymbol{\Delta}_{i}^{1 / 2}\right\}=\alpha \mathbf{I}_{N} \\
\mathrm{E}\left\{\mathbf{Y}_{i}^{\mathrm{H}} \mathbf{X}_{i}^{\mathrm{H}} \boldsymbol{\Delta}_{i} \mathbf{X}_{i} \mathbf{Y}_{i}\right\}=(M-N) \mathbf{I}_{N} \\
\mathrm{E}\left\{\mathbf{S}_{i}^{\mathrm{H}} \mathbf{S}_{i}\right\}=\frac{1}{M-N}\left(\mathbf{I}_{M}-\hat{\mathbf{H}}_{i} \hat{\mathbf{H}}_{i}^{\mathrm{H}}\right)
\end{gathered}
$$




$$
\mathrm{E}\left\{\mathbf{Z}_{i}^{\mathrm{H}} \boldsymbol{\Delta}_{i} \mathbf{Z}_{i}\right\}=\frac{D M}{N} \mathbf{I}_{N}
$$

\section{Beamforming Algorithm based on MMSE}

According to two parts, described earlier in this section, first of all, the optimization function is defined as the primary user interference power conditions, restricted and given to minimize the mean square error of the optimization goal, as the concrete mathematical expression

$$
\begin{array}{ll} 
& \min _{\left\{\mathbf{F}_{1}, \cdots \mathbf{F}_{\mathrm{K}}\right\}\left\{\mathbf{Q}_{1} \cdots \mathbf{Q}_{\mathrm{K}}\right\}} \sum_{i=1}^{\mathrm{K}} \operatorname{Tr}\left(\varepsilon_{i}\right) \\
\text { s.t. } & p_{i} \leq P_{k} \\
& \operatorname{Tr}\left(\sum_{i=1}^{K} \mathbf{F H}_{i} \mathbf{H}_{i}^{\mathrm{H}} \mathbf{F}^{\mathrm{H}} P_{i}+\mathbf{F} \mathbf{F}^{\mathrm{H}} \sigma^{2}\right) \leq P_{R} \\
& \sum_{i=1}^{K}\left\|\mathbf{G}_{i}\right\|^{2} P_{i} \leq \eta, \\
& \operatorname{Tr}\left(\sum_{i=1}^{K} \mathbf{G}_{i} \mathbf{F H}_{i} \mathbf{H}_{i}^{\mathrm{H}} \mathbf{F}^{\mathrm{H}} \mathbf{G}_{i}^{\mathrm{H}} P_{i}+\mathbf{G}_{i} \mathbf{F} \mathbf{F}^{\mathrm{H}} \mathbf{G}_{i}^{\mathrm{H}} \sigma^{2}\right) \leq \eta
\end{array}
$$

$\varepsilon_{i}$ denotes the Mean square error (mse) of second user $i(i=1, \cdots, K)$, for its expression

$$
\varepsilon_{i}\left(\mathbf{F}_{i}, \mathbf{Q}_{i}\right)=\left(\mathbf{Q}_{i} \boldsymbol{y}_{i}-\boldsymbol{X}_{i}\right)\left(\boldsymbol{y}_{i}^{\mathrm{H}} \mathbf{Q}_{i}^{\mathrm{H}}-\boldsymbol{X}_{i}^{\mathrm{H}}\right)
$$

For limited feedback system, the relay side only know quantitative channel state information $\mathbf{H}_{i}$, the simple direct approach is the base station will quantify the CDI considered accurate CSI, precoding ${ }^{[10]}$, and this method does not consider the influence of quantization error. In order to consider the effect of quantization error on the system, based on the average MSE in order to optimize the function $\mathbf{B}_{k}$ and $\mathbf{A}_{k}$, the joint optimization, and so robust MMSE precoding optimization problems can be summed up in:

$$
\begin{aligned}
& \min _{\left\{\mathbf{F}_{1}, \cdots, \mathbf{F}_{\mathrm{K}}\right\}\left\{\mathbf{Q}_{1} \cdots \mathbf{Q}_{\mathrm{K}}\right\}} \sum_{k=1}^{\mathrm{K}} \operatorname{Tr}\left\{E_{\mathbf{H}_{i} \mid \hat{\mathbf{H}}_{i}}\left[\varepsilon_{i}\left(\mathbf{F}_{i}, \mathbf{Q}_{i}\right)\right]\right\} \\
& \text { s.t. } \quad p_{i} \leq P_{i} \\
& \quad \operatorname{Tr}\left(\sum_{i=1}^{K} \mathbf{F H}_{i} \mathbf{H}_{i}^{\mathrm{H}} \mathbf{F}^{\mathrm{H}} P_{i}+\mathbf{F} \mathbf{F}^{\mathrm{H}} \sigma^{2}\right) \leq P_{R} \\
& \sum_{i=1}^{K}\left\|\mathbf{G}_{i}\right\|^{2} P_{i} \leq \eta, \\
& \quad \operatorname{Tr}\left(\sum_{i=1}^{K} \mathbf{G}_{i} \mathbf{F} \mathbf{H}_{i} \mathbf{H}_{i}^{\mathrm{H}} \mathbf{F}^{\mathrm{H}} \mathbf{G}_{i}^{\mathrm{H}} P_{i}+\mathbf{G}_{i} \mathbf{F} \mathbf{F}^{\mathrm{H}} \mathbf{G}_{i}^{\mathrm{H}} \sigma^{2}\right) \leq \eta
\end{aligned}
$$

From optimizing functional (20) can be obtained, for $\mathbf{F}_{i}$ or $\mathbf{Q}_{i}, \varepsilon_{i}\left(\mathbf{F}_{i}, \mathbf{Q}_{i}\right)$ as the convex function, and to send a beam forming matrix $\mathbf{F}_{i}$ and receive beam forming matrix $\mathbf{Q}_{i}$ of 
joint function, the optimization of objective function is convex function. According to the nonlinear optimization theory, in this paper, using Lagrange multiplier method to solve the optimization problem, and then puts forward an effective iterative optimization algorithm. Lagrangian dual objective function is:

$$
\begin{aligned}
L\left(\mathbf{F}_{i} ; \mathbf{Q}_{i} ; \lambda_{i} ; v_{i} ; \theta_{i} ; \gamma_{i}\right) & =\sum_{k=1}^{\mathrm{K}} \operatorname{Tr}\left\{E_{\mathbf{H}_{i} \mid \hat{H}_{i}}\left[\varepsilon_{i}\left(\mathbf{F}_{i}, \mathbf{Q}_{i}\right)\right]\right\}-\sum_{k=1}^{K} \lambda_{k}\left[p_{i}-P_{i}\right] \\
& -v_{i}\left[\operatorname{Tr}\left(\sum_{i=1}^{K} \mathbf{F} \mathbf{G}_{i} \mathbf{G}_{i}^{\mathrm{H}} \mathbf{F}^{\mathrm{H}} P_{i}+\mathbf{F} \mathbf{F}^{\mathrm{H}} \sigma^{2}\right)-P_{R}\right] \\
& -\theta_{i}\left[\sum_{i=1}^{K}\left\|\mathbf{G}_{i}\right\|^{2} P_{i}-\eta\right] \\
& -\gamma_{i}\left[\operatorname{Tr}\left(\sum_{i=1}^{K} \mathbf{G}_{i} \mathbf{F} \mathbf{H}_{i} \mathbf{H}_{i}^{\mathrm{H}} \mathbf{F}^{\mathrm{H}} \mathbf{G}_{i}^{\mathrm{H}} P_{i}+\mathbf{G}_{i} \mathbf{F} \mathbf{F}^{\mathrm{H}} \mathbf{G}_{i}^{\mathrm{H}} \sigma^{2}\right)-\eta\right]
\end{aligned}
$$

Which $\lambda_{i} ; v_{i} ; \theta_{i} ; \gamma_{i} \in R$ is a Lagrange multiplier, the application Karush - Kuhn Tucher (KKT) conditions, the summation $L\left(\mathbf{F}_{i} ; \mathbf{Q}_{i} ; \lambda_{i} ; v_{i} ; \theta_{i} ; \gamma_{i}\right)$ espectively derivatives $\mathbf{F}_{i}$ and $\mathbf{Q}_{i}$ can be obtained:

$$
\begin{aligned}
& \quad \frac{\partial L\left(\mathbf{F}_{i} ; \mathbf{Q}_{i} ; \lambda_{i} ; v_{i} ; \theta_{i} ; \gamma_{i}\right)}{\partial \mathbf{F}_{i}^{*}}=\left[M N(N-D)-\frac{1}{(M-N) N} \sum_{i=1}^{i} \hat{\mathbf{H}}_{i}^{\mathrm{H}} \mathbf{Q}_{i}^{\mathrm{H}} \mathbf{Q}_{i} \hat{\mathbf{H}}_{i} \mathbf{F}_{i}+\frac{M^{2} D}{(M-N) N} \sum_{i=1}^{K} \mathbf{Q}_{i}^{\mathrm{H}} \mathbf{Q}_{i} \mathbf{F}_{i}\right. \\
& \quad-(M-N) \sum_{k=1}^{K} \hat{\mathbf{H}}_{i}^{\mathrm{H}} \mathbf{Q}_{i}^{\mathrm{H}} \mathbf{Q}_{i} \hat{\mathbf{H}}_{i} \mathbf{F}_{i}-\frac{D M^{2}}{M-N} \sum_{i=1}^{K} \mathbf{Q}_{i}^{\mathrm{H}} \mathbf{Q}_{i} \mathbf{F}_{i}+\frac{D M}{M-N} \sum_{k=1}^{K} \mathbf{Q}_{i}^{\mathrm{H}} \mathbf{Q}_{i} \hat{\mathbf{H}}_{i} \mathbf{F}_{i}-\alpha \sum_{i=1}^{K} \hat{\mathbf{H}}_{i} \mathbf{Q}_{i}+\sum_{i=1}^{K} v_{i} \mathbf{F}_{i}+\sum_{i=1}^{K} \gamma_{\mathbf{i}_{i}} \mathbf{Q}_{i}^{\mathrm{H}} \mathbf{Q}_{i} \mathbf{F}_{i} \\
& \frac{\partial L\left(\mathbf{F}_{i} ; \mathbf{Q}_{i} ; \lambda_{i} ; v_{i} ; \theta_{i} ; \gamma_{i}\right)}{\partial \mathbf{Q}_{i}^{*}}=\left[M N(N-D)-\frac{1}{(M-N) N}\right] \sum_{i=1}^{K} \mathbf{Q}_{i} \hat{\mathbf{H}_{i}} \mathbf{F}_{i} \mathbf{F}_{i}^{\mathrm{H}} \hat{\mathbf{H}}_{i}^{\mathrm{H}}+\frac{M^{2} D}{(M-N) N} \sum_{i=1}^{K} \mathbf{Q}_{i} \mathbf{F}_{i} \mathbf{F}_{i}^{\mathrm{H}}+(M-N) N P \sum_{i=1}^{K} \mathbf{Q}_{i} \hat{\mathbf{H}}_{i} \hat{\mathbf{H}}_{i}^{\mathrm{H}} \\
& +\frac{D M P}{M-N} \sum_{i=1}^{K}\left(\mathbf{Q}_{i}-\mathbf{Q}_{i} \hat{\mathbf{H}}_{i} \hat{\mathbf{H}}_{i}^{\mathrm{H}}\right)+\sum_{i=1}^{K} \mathbf{Q}_{i}-(M-N) \sum_{k=1}^{K} \mathbf{Q}_{i} \hat{\mathbf{H}}_{i} \mathbf{F}_{i} \mathbf{F}_{i}^{\mathrm{H}} \hat{\mathbf{H}}_{i}^{\mathrm{H}}-\frac{D M^{2}}{M-N} \sum_{k=1}^{K} \mathbf{Q}_{i} \mathbf{F}_{i} \mathbf{F}_{i}^{\mathrm{H}}+\frac{D M}{M-N} \sum_{i=1}^{K} \mathbf{Q}_{i} \hat{\mathbf{H}}_{i} \mathbf{F}_{i} \mathbf{F}_{i}^{\mathrm{H}} \hat{\mathbf{H}}_{i}^{\mathrm{H}}-\alpha \sum_{i=1}^{K} \mathbf{F}_{i} \hat{\mathbf{H}}_{i} \\
& =\mathbf{0}
\end{aligned}
$$

By type (24) and (25), you can see that each time the user sends a beam forming matrix $\left\{\mathbf{F}_{i}\right\}$ and optimal receiving beam forming matrix $\left\{\mathbf{Q}_{i}\right\}$ are involved. In this paper, matrix iterative calculation $\left\{\mathbf{F}_{i}\right\}$ and receive $\left\{\mathbf{Q}_{i}\right\}$

Proposed MMSE algorithm

1. Set the iteration variable $n=0$. Initialization $\mathbf{F}_{i}(0), i=1, \cdots K$.

2. $n \leftarrow n+1$, update receiving matrix $\mathbf{Q}_{i}$ : see type (25)

3. Calculate and send update matrix $\mathbf{F}_{i}$ : see type (24)

4. Repeat step 2 and step 3 until convergence 


\section{Beamforming Matrix Initialization Problem Solving:}

Method 1: Carried out on the channel matrix $\hat{\mathbf{H}}_{i}$ singular value decomposition, the normalized right as sending a beam forming matrix singular matrix $\mathbf{F}_{i}(0), i=1, \cdots K$.

Method 2: Randomly generated a zero mean and unit variance of the gaussian matrix as the initialization matrix sends a beam forming $\mathbf{F}_{i}(0), i=1, \cdots K$.

\section{Algorithm Convergence Problem}

By type (24), $\operatorname{MSE}\left(\mathbf{F}_{k}(n+1), \mathbf{Q}_{k}(n)\right) \leq \operatorname{MSE}\left(\mathbf{F}_{k}(n), \mathbf{Q}_{k}(n)\right)$ and also, by type (25), $\operatorname{MSE}\left(\mathbf{F}_{k}(n+1), \mathbf{Q}_{k}(n+1)\right) \leq \operatorname{MSE}\left(\mathbf{F}_{k}(n+1), \mathbf{Q}_{k}(n)\right)$ and finally you can get $\operatorname{MSE}\left(\mathbf{F}_{k}(n+1), \mathbf{Q}_{k}(n+1)\right) \leq \operatorname{MSE}\left(\mathbf{F}_{k}(n+1), \mathbf{Q}_{k}(n+1)\right)$

Prove completely

\section{Simulation and Results}

In this part, using the computer simulation to show the advanced nature of the proposed scheme; In order to simplify the simulation, this paper argues that the user is equal to two-way relay distance. Do not break general, RS and RS is the user to the primary user distance to the user from two times and three times. In this simulation, $M=8, N=2, N=2$, User and set a time equal to the maximum power of the relay sends, i.e $P_{R}=P_{k}=P_{t}$. In the simulation, set up different stages of boundaries are the primary user interference. In limited feedback mechanism, the simulation USES the stochastic high 'flow pattern quantitative code, the number of iterations for 8 times. In order to compare the feedback scheme under the condition of superiority, this paper simulates the feedback bits for the 6 bit and 12 bit. In order to compare convenience, this article proposed solutions respectively compared with the following three schemes:

Solution 1: optimal power allocation scheme, the legend of Figure 2 and Figure 3 recorded as "[9]";

Solution 2: to maximize user system transmission rate, Figure 2 and Figure 3 in the legend of recorded as "[10] ";

Solution 3: to maximize user system transmission rate and the optimal power allocation optimization, the legend of Figure 2 and Figure 3 as a "joint".

Figure 2 in the user's average signal-to-noise ratio $(0 \square 30 \mathrm{~dB})$, the maximum mutual information of the user's system, left feedback bits for the 6 bit is the case, as can be seen from the left, the proposed scheme and optimal scheme 3, $2 \mathrm{~dB}$ gain, the main reason for CSI scheme 3 is based on the RS obtain complete under the condition of the optimal solution, without considering quantization error exists, from another Angle and you can see, quantitative error affect user transmission rate. Right due to the increased number of bits in the feedback to the 12 bit, RS for CSI is more accurate, the proposed scheme advantages are more obvious, you can see there is a gain of $2.5 \mathrm{~dB}$. As can be seen from the two figure of Figure 2, the proposed scheme is effective to overcome the influence of quantization error to the user transmission rate. 

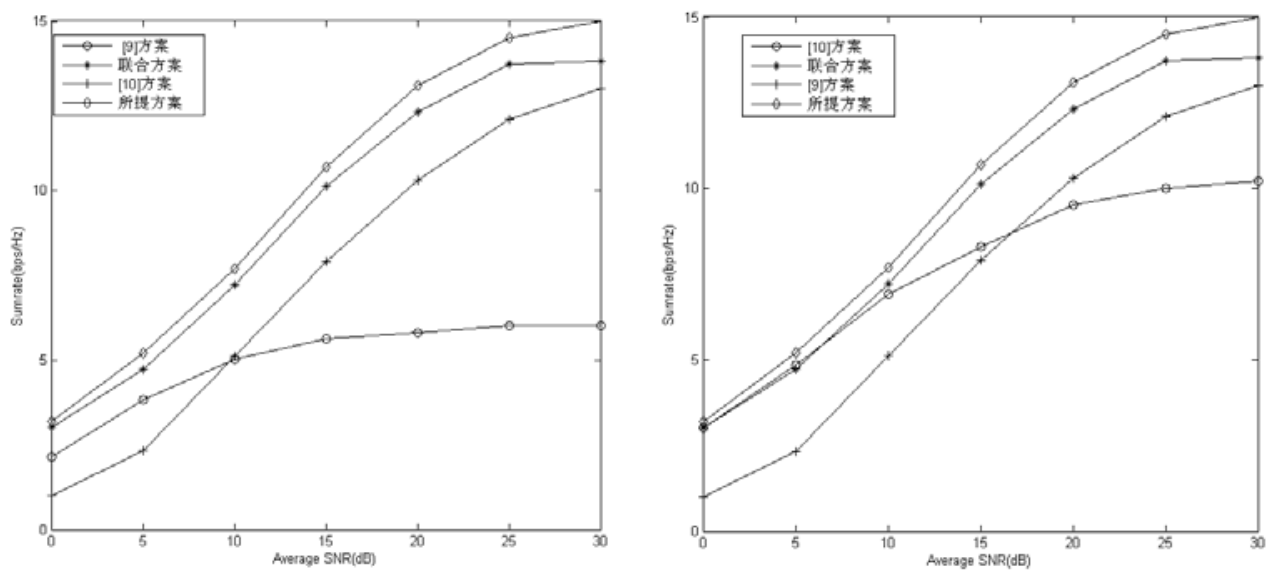

Figure 2. SU Transmission Rate Comparison

Figure 3 in the same time the user's average signal-to-noise ratio ( $0 \square 30 d B$ ), the bit error performance of four kinds of schemes are compared, left for feedback bits for 6 bit, the BER curves, we can find that, due to the optimization criteria for the MMSE, so its BER performance advantage is more obvious, the other three kinds of solution with the increase of SNR, the BER curve does not decline, while the proposed scheme has a good BER and convergence with the convergence of mean square error (MSE) have equivalence, from the perspective of the simulation prove that the convergence of the algorithm. Also, the picture on the right of feedback bits of 12 bit, its performance advantages more apparent.
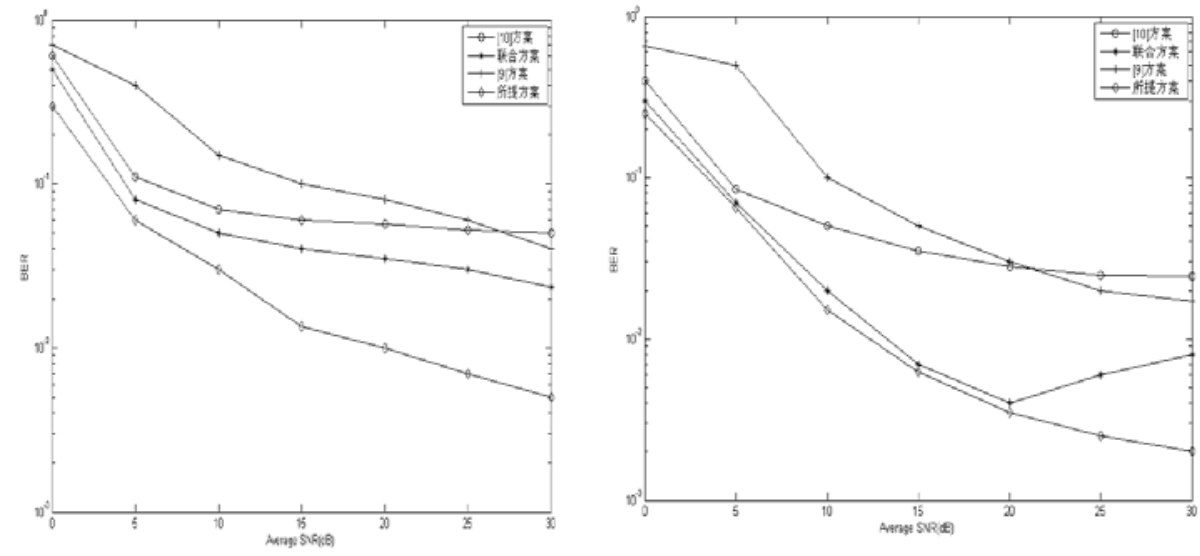

Figure 3. BER Comparison

\section{Conclusion}

The paper studied under the condition of limited feedback, under the condition of ensure the main user QoS, two-way relay by sending a beam forming matrix and the second user receiving beam forming joint processing matrix, to eliminate the interference between multiple users, and effectively suppress the interference to primary user, and the simulation results show that the proposed in this paper, based on the MMSE and the beam forming joint scheme effectively improves the user system of mutual information, and reduces the bit error rate. 


\section{References}

[1] M. Zhao, C. Yin and X. Wang, "A novel dynamic spectrum access algorithm for cognitive radio networks", Communications and Networks, (2013), pp. 38-44.

[2] T. Li, N. Mandayam and A. B. Reznik, "A Framework for Distributed Resource Allocation and Admission Control in a Cognitive Digital Home”, IEEE Transactions on Wireless Communications, vol. 12, no. 3, (2013), pp. 984-995.

[3] S. Zheng, P. Kam, Y. Liang and Y. Zeng, "Spectrum Sensing for Digital Primary Signals in cognitive Radio: A Bayesian Approach for Maximizing Spectrum Utilization”, IEEE Transactions on Wireless Communications, vol. 99, no. 99, (2013), pp. 1-9.

[4] B. A. Bastami and E. A. Saberinia, "Practical Multibit Data Combining Strategy for Cooperative Spectrum Sensing”, IEEE Transactions on Vehicular Technology, vol. 62, no. 1, (2013), pp. 384-389.

[5] D. Gunduz, E. Erkip, A. Goldsmith and H. V. Poor, "Reliable Joint Source-Channel Cooperative Transmission Over Relay Networks”, IEEE Transactions on Information Theory, vol. 9, no. 4, (2013), pp. 2442-2458.

[6] X. Liang, S. Jin, X. Gao and K.-K. Wong, "Outage Performance for Decode-and-Forward Two-Way Relay Network with Multiple Interferers and Noisy Relay”, IEEE Transactions on Communications, vol. 61, no. 2, (2013), pp. 521-531.

[7] Y. Huang, L. Yang and M. Bengtsson, "A limited feedback joint precoding for amplify-and-forward relaying”, IEEE Transactions on Signal Processing, vol. 58, no. 3, (2010), pp. 1347-1357.

[8] W. Xu, X. Dong and W. Lu, "MIMO relaying broadcast channels with linear precoding and quantized channel state information feedback”, IEEE Transactions on Signal Processing, vol. 58, no. 10, (2010), pp. 5223-5245.

[9] R. Wang, M. Tao and Y. Liu, "Optimal Linear Transceiver Designs for Cognitive Two Way Relay Networks”, IEEE Transactions on Signal Processing, vol. 61, no. 4, (2013), pp. 992-1005.

[10] S. H. Safavi, M. Ardebilipour and S. Salari, "Relay Beamforming in Cognitive Two-Way Networks with Imperfect Channel State Information”, IEEE Wireless Communications Letters, vol. 1, no. 4, (2012), pp. 344-347.

[11] K. Jitvanichphaibool, Y. Liang and R. Zhang, "Beamforming and Power Control for Multi-Antenna Cognitive Two-Way Relaying”, IEEE Wireless Communications and Net-working Conference, WCNC 2009, (2009).

[12] T. Wang, L. Song, H. Zhu and B. Li, "Improve secure communications in cognitive two-way relay networks using sequential second price auction”, IEEE Symposium on New Frontiers in Dynamic Spectrum Access Networks (DySPAN), (2011), pp. 308-315.

[13] N. Ravindran and N. Jindal, "Limited feedback-based block diagonalization for the MIMO broadcast channel”, IEEE Transactions on selected areas in communications, vol. 26, no. 8, (2008), pp. 1473-1482.

[14] A. Sharma and A. Chaturvedi, "Gradient Descent Feed Forward Neural Networks for Forecasting the Trajectories, IJAST, vol. 34, (2011) September, pp. 83-88.

[15] S. Lee, "Security Issues of Power Line Multi-Home Networks for Seamless", IJAST, vol. 37, (2011) December, pp. 141-146.

[16] K.-I. Kim, “A Simulation Study for Typical Routing Protocols in Aircraft Ad Hoc Networks”, IJSEIA, vol. 7, no. 2, (2013) March, pp. 227-234.

[17] R. Bhimanpallewar and P. Metkewar, “Aggregation Query Processing in P2P Networks”, IJDTA, vol. 5, no. 3, (2012) September, pp. 55-72.

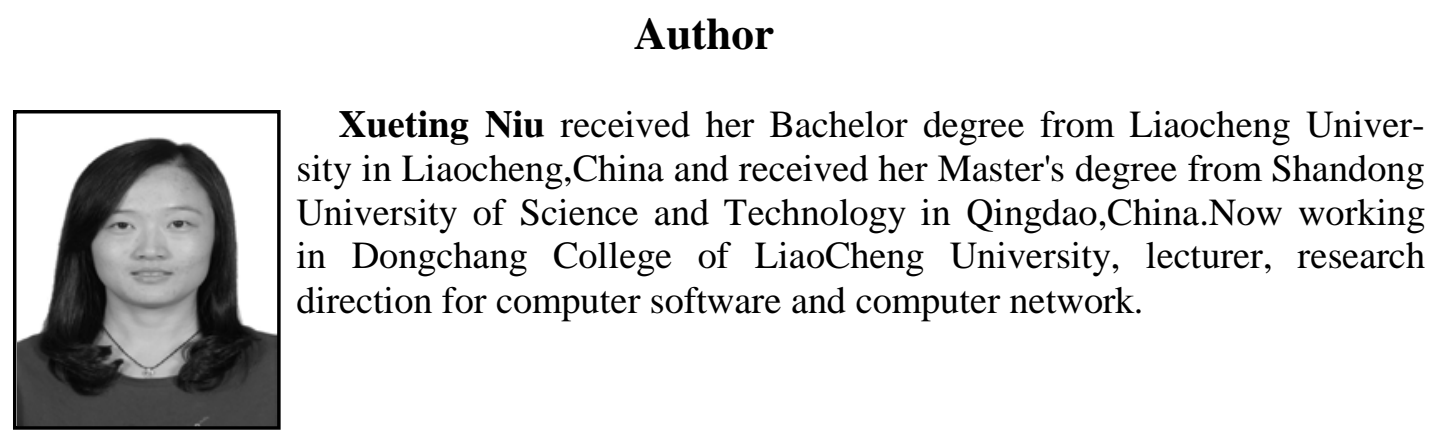


International Journal of Future Generation Communication and Networking Vol.6, No.5 (2013) 